\title{
IMPACTO DE LA CRISIS FINANCIERA INTERNACIONAL EN EL SECTOR TEXTIL PERUANO
}

Elena Bautista F. (*)

E-mail: ebautistaf@unmsm.edu.pe

Allan Vargas G. (*)

E-mail:avargasg@unmsm.edu.pe

Víctor Castro M. (*****)

E-mail:vcastrom@unmsm.edu.pe

"La mejor estructura no garantizará los resultados ni el rendimiento. Pero la estructura equivocada es una garantía de fracaso".

Peter Drucker

\begin{abstract}
RESUMEN
La industria textil peruana entró en desaceleración a partir del 2009, presentando la mayor caída registrada al menos en los últimos 15 años. El derrumbe de la demanda externa en nuestro mercado de destino más importante (Estados Unidos) debido a la crisis internacional, y una agresiva competencia China en este mercado explican, en gran parte, estos resultados. Las exportaciones del sector textilconfecciones han cerrado el 2009 en US\$ 1,550 millones, 23\% debajo del nivel alcanzado en el 2008. El segmento más afectado fue el de confecciones que se habría contraído $25 \%$ mientras el de textiles (fibras, hilados y tejidos) lo habría hecho en $15 \%$.

En los últimos meses del 2009 se presentó una tendencia creciente en los costos de producción (hilados de algodón) que encarecen los productos finales y restan competitividad frente a los productos asiáticos, caracterizados por ser más económicos pero de menor calidad.

El año 2010, el Ministerio de Comercio Exterior y Turismo formuló importantes propuestas para el sector textil peruano: fortalecimiento de la cadena de algodón-hilado-textil-prenda, desarrollo de políticas que potencien las ventajas comparativas del sector, utilización del algodón peruano de alta

(*) Estudios de Doctorado. Egresada de Maestría en Administración. Licenciada en Administración. Profesora Asociada de la Facultad de Administración de la UNMSM.

(**) Magister en Administración. Licenciado en Administración. Profesor Auxiliar de la Facultad de Administración de la UNMSM.

(***) Estudios de Doctorado. Egresado de Maestría en Administración. Licenciado en Administración. Profesor Asociado de la Facultad de Administración de la UNMSM.
\end{abstract}


calidad y costo razonable, capacitación y entrenamiento a lo largo de toda la cadena, y promoción de la innovación tecnológica, tanto para la producción y adaptación de nuevas semillas como para el desarrollo de hilados, textiles y confecciones que se consoliden en el nicho de mercado de alta calidad.

Para el año 2010, se ha proyectado un crecimiento de la economía peruana de un $6.8 \%$ que se explica por la recuperación de la economía internacional, liderada por los países emergentes y, especialmente, por el fuerte dinamismo de la demanda interna local en el primer semestre del 2010. Sin embargo, para el año 2011, se mantiene la proyección de crecimiento de la economía peruana de $5 \%$ debido a la incertidumbre respecto del contexto internacional y a la extinción de los factores temporales que impulsaron el crecimiento en el 2010, como la recomposición de inventarios, la política económica expansiva y el efecto rebote. De no materializarse una recaída de la economía mundial, el Perú crecerá a una tasa sostenida cerca al 6\% para los años 2012 y 2013 de la mano de importantes proyectos de inversión privada, debido también a un mayor protagonismo de los países emergentes, fuertes demandantes de commodities.

Palabras Clave: Perspectivas de largo plazo, Exportaciones de textiles, Impacto económico en la economía nacional, Evaluación estratégica.

\section{ABSTRACT}

Peruvian textile industry slows down from 2009 showing the largest decline recorded in at least 15 years. The collapse of foreign demand in our most important target market -USA, due to international crisis, and the aggressive competition of China in this market.

Exports of textile and apparel sector have closed 2009 at US\$ 1,550 million, 23\% below the level reached in 2008 . The most affected segment was the garment that would have made $25 \%$, meanwhile textiles (fibers, yarns and fabrics) would have 15\%.

In the last months of 2009 there was a growing trend in production costs (cotton yarn) to make finished products more expensive and less competitive against Asian products, characterized by being cheaper but lower quality.

In 2010, the Ministry of Foreign Trade and Tourism made important proposals for the Peruvian textile sector: strengthening the chain of cotton-yarn-textile-garment; developing policies to improve the sector's comparative advantages; use of high-quality Peruvian cotton and reasonable cost: training throughout the chain, and promotion of technological innovation, both for production and adaptation of new seeds to the development of yarn, textiles and garments that are consolidated in the market of high quality.

For the year 2010, it has projected a growth of the Peruvian economy by $6.8 \%$ and is attributable to a recovery in the international economy, led by emerging economies, and especially by the strong dynamism of domestic demand at first half of 2010. However, for the year 2011 Peruvian economy will maintain the growth projection of 5\% due to the uncertainty in the international context and termination of temporary factors that boosted growth in 2010 , as the rebuilding of inventories, expansionary economic policy and the rebound effect. If the world economy will not fall down, Peru will grow at rates around 6\% for the years 2012 to 2013 for a major private investment projects, also a greater role of emerging countries, strong commodity applicants.

Key Words: Long-Term Prospects, Exports of textiles, Economic impact on national economy, Strategic Assessment.

\section{INTRODUCCIÓN}

La inclusión de los textiles y de las confecciones en el universo de productos beneficiados por la Ley de Preferencias Arancelarias Andinas (ATPDEA), a finales del 2002, se tradujo en tasas de crecimiento promedio anual de la industria textil de $10.3 \%$ en el período 2002-2007, que contribuyó en gran medida a materializar el boom exportador de los últimos años de la industria de confecciones. A partir del 2007 la industria empezó a contraerse, afectada por la masiva importación de productos que ingresaban al país con menores precios. En el 2008 la caída de la producción textil se acentuó con la reducción de las exportaciones de confecciones (principal demandante de los productos textiles) provocada por la disminución 
de la demanda de Estados Unidos en el marco de la crisis internacional.

A fin de hacer frente a la competencia, muchas veces desleal de las confecciones, fibras, hilados y tejidos importados a menores precios que los nacionales, se aplicaron mecanismos de protección. En agosto de 1995 se decidió la aplicación de derechos antidumping a las importaciones de tejidos de algodón y tejidos mixtos procedentes de China, los cuales fueron ratificados en el 2002. En marzo del 2004 el Instituto Nacional de Defensa de la Competencia y de la Protección de la Propiedad Intelectual (INDECOPI) dispuso la aplicación de derechos antidumping a la importación de tejidos planos de ligamento tafetán, popelina, poliéster, algodón, estampados crudos, blanqueados, teñidos o con hilados de diferentes colores originarios de Pakistán. En el 2006 se estableció que los tejidos de denim originarios de Brasil pagarán derechos antidumping.

\section{Estructura interna}

La industria textil comprende la producción de fibras, hilados y tejidos, en nueve categorías de productos: fibras naturales, fibras artificiales, tejidos planos, tejidos de punto, tejidos industriales, revestimientos para pisos, productos para el hogar, textiles no tejidos y sogas (cuerdas) (ver Cuadro $\mathrm{N}^{\circ} 1$ ).

El 60\% de la industria corresponde a la fabricación de textiles a base de algodón, el 35\% a fibras sintéticas y el $5 \%$ a textiles con fibras de origen animal. La producción se destina en su mayor proporción a atender la demanda de la industria de confecciones orientada a la exportación. Existen cerca de 400 empresas en los rubros de hilandería y tejeduría, y la mayoría son pequeñas y microempresas.

\section{Cadena productiva textil}

La principal materia prima utilizada por la industria textil es el algodón, que por sus características especiales es considerado entre los de mejor calidad en el mundo, principalmente por la longitud de la fibra -extra larga- que permite fabricar hilados y telas muy finas, que son altamente cotizados en el mercado internacional. Otras materias primas empleadas en la industria textil son la lana de alpaca, pelos de animal y las fibras sintéticas.

En el sector textil existen cinco líneas principales de productos agrupados en los siguientes sub-sectores:

- Textil algodonero.

- Textil lanero y alpaquero (incluye hilados y tejidos).

- Textil fibras sintéticas.

- Confección de prendas de vestir.

- Confecciones de textiles no prendas de vestir.

- Otros textiles.

Cuadro $\mathbf{N}^{\circ}$ 1. Principales flujos del sector textil

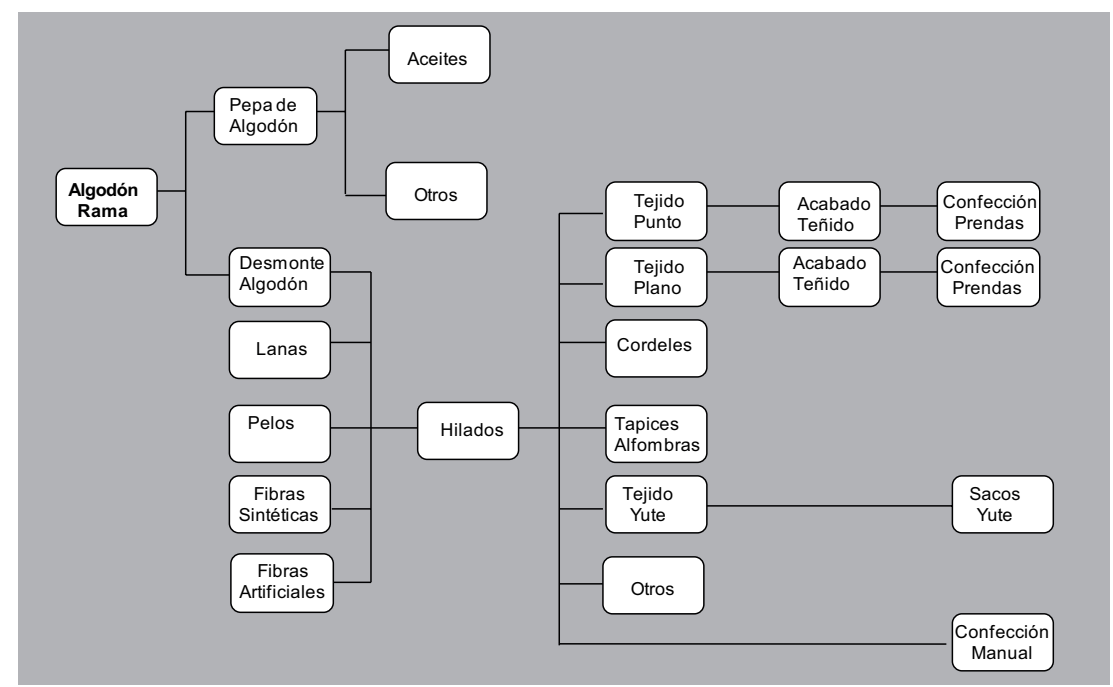

Fuente: Corporación Financiera de Desarrollo- COFIDE

1 Ver Maximixe (2009). 
Importaciones y exportación mundiales de tejidos de algodón

En el 2008, las importaciones mundiales de tejidos de algodón sumaron US\$ 9,197 millones, $1.2 \%$ más respecto al 2007 (ver Gráfico $\mathrm{N}^{\circ} 1$ ). Las compras de Túnez crecieron 3.2\%, las de Bangladesh, en 369\%, y las de Vietnam, en $18.4 \%$. Los principales importadores de tejidos de algodón fueron Hong Kong, con 11.7\% del total; China, con 10.8\%; Túnez, que alcanza el 7\% y Bangladesh, con el $6.7 \%$. Perú se ubicó en el puesto 49 del ranking de importadores. Las importaciones de Latinoamérica y El Caribe sumaron US\$ 1,002 millones en el 2008, 0.01\% más que en el 2007. Las importaciones de Nicaragua crecieron $3,254.2 \%$; las de Honduras, 808\%; Bolivia, 378\% y Brasil, 137.5\%.

Las exportaciones mundiales de tejidos de algodón sumaron US\$ 9,142 millones en el 2008 (ver Gráfico $\mathrm{N}^{\circ} 2$ ), esto es $4.3 \%$ menos que el 2007 debido a las menores exportaciones de Hong Kong $(-10.1 \%)$, Italia $(-5.1 \%)$, Estados Unidos (-7.4\%), entre otros. China lideró las exportaciones con $24.6 \%$ de las mismas, seguida por Hong Kong con $15.7 \%$ e Italia con 9.6\%. Perú ocupó el puesto 34 en el ranking de exportadores mundiales.

Gráfico $\mathbf{N}^{\circ}$ 1. Evolución de las importaciones mundiales de tejidos de algodón (en millones de US\$).

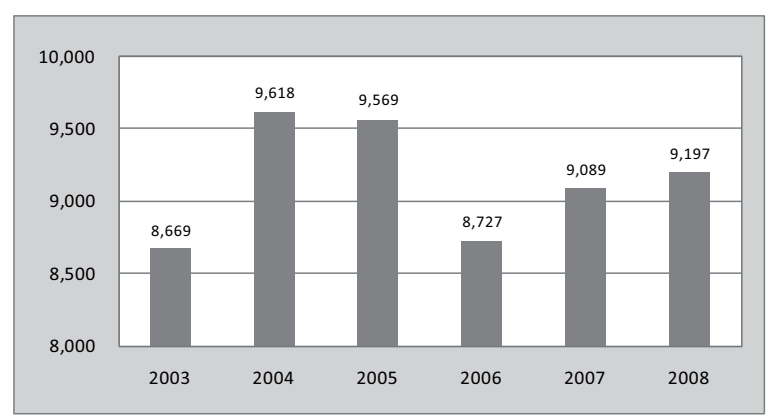

Fuente: Ministerio de la Producción

Gráfico $N^{\circ} 2$. Evolución de las exportaciones mundiales de tejidos de algodón (en millones de US\$).

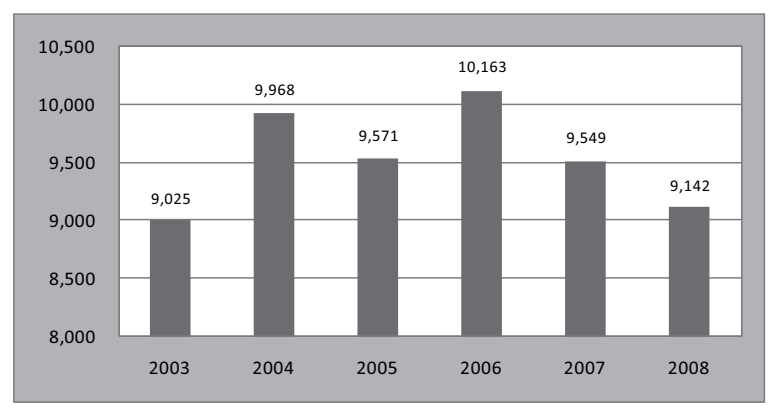

Fuente: Ministerio de la Producción

\section{Caída de la producción textil en el Perú}

El año 2009, la industria textil se contrajo $28 \%$ respecto al 2008 , debido a que su principal demandante, la industria de confecciones, caería $29.2 \%$ ante el retroceso de sus exportaciones $(-20.8 \%)$, por la menor demanda de Estados Unidos y Venezuela en el contexto de la crisis internacional. También se vería afectada por la sustitución de los textiles nacionales por productos de origen importado, especialmente en el rubro hilados. Para el 2010 se proyecta un crecimiento de la actividad textil de $8.7 \%$ a partir de la recuperación de los pedidos de confecciones de Estados Unidos, en línea con la reactivación de su economía y de la economía mundial.

Entre enero y julio del 2009 la industria textil cayó $26.9 \%$ respecto a similar período del año pasado, debido a la contracción de la industria de confecciones (-32.9\%) que, en promedio, ha operado al $38 \%$ de su capacidad instalada, en perjuicio de sus márgenes de rentabilidad, originando un incremento de los costos fijos en su estructura de costos.

\section{Caída de las exportaciones textiles en el Perú (no incluyen confecciones)}

Al cierre del 2009 las exportaciones de textiles sumarían US\$ 333.4 millones, $10.6 \%$ menos que en el 2008. Los envíos de fibras caerían $31.7 \%$ y los de hilados $26.1 \%$, en un escenario internacional adverso. Sin embargo, las exportaciones de tejidos se incrementarían en $7.7 \%$.

A julio del 2009 las exportaciones de textiles cayeron $15.3 \%$ (hasta US\$ 184.4 millones)t respecto al mismo período del año pasado. Los envíos de fibras retrocedieron $44.9 \%$ y los de hilados $27.5 \%$, mientras que los de tejidos avanzaron $10.7 \%$ debido a los mayores envíos a Venezuela y Chile.

Para el 2010 se espera que las exportaciones de textiles se recuperen y crezcan $9.6 \%$, por la reactivación de la demanda de los principales países demandantes de textiles peruanos, especialmente de Estados Unidos.

En el período 2003-2008 las exportaciones crecieron a una tasa promedio anual de $17.1 \%$, favorecidas por la ratificación y ampliación de la Ley de Preferencias Arancelarias Andinas (ATPDEA) en el 2002, que permitió el ingreso de los productos peruanos libres de aranceles al mercado estadounidense. 
Gráfico $\mathrm{N}^{\circ}$ 3. Exportaciones textiles en el Perú (en millones de US\$).

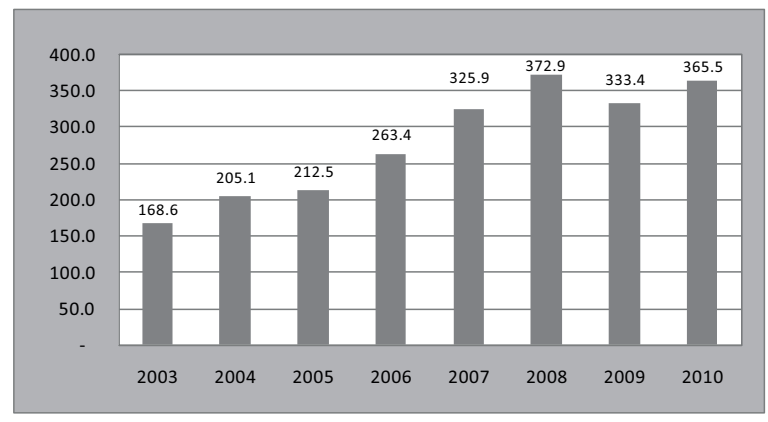

Fuente: Superintendencia Nacional de Administración Tributaria (SUNAT).

En el 2008 las exportaciones textiles sumaron US\$ 372.9 millones, $14.4 \%$ más respecto al 2007, por mayores envíos de tejidos (34.2\%) e hilados (7.5\%). El valor de las exportaciones de tejidos fue el $49.7 \%$ de las exportaciones textiles, seguido por hilados (27.4\% del total) y fibras $822.8 \%)^{2}$.

\section{Exportaciones de textiles-confecciones por empresas}

Las exportaciones de las 25 principales empresas exportadoras de textiles cayeron $19 \%$ en el periodo enero-octubre del 2009 respecto del mismo periodo del 2008 (ver Cuadro $\mathrm{N}^{\circ}$ 2). Solo cuatro empresas de esta lista mostraron tasas de crecimiento en sus niveles exportados y dichos resultados se explican básicamente por ser nuevas empresas exportadoras que nacieron por la desaparición o desmembramiento de otras unidades productivas.

\section{Las salvaguardias y la protección de la industria textil peruana}

La Comisión Multisectorial, integrada por el Ministerio de Economía y Finanzas (MEF) y el Ministerio de Comercio Exterior, Turismo y Producción (MINCETUR), desestimó la recomendación de la Comisión de Fiscalización de Dumping y Subsidios del INDECOPI y decidió la no aplicación de salvaguardias a la importación de hilados procedentes de la India, aludiendo falta de información respecto a que los productos nacionales eran iguales o directamente competidores.
Cuadro $\mathbf{N}^{\circ} 2$. Exportaciones de textiles-confecciones según empresas (En millones de US\$)

\begin{tabular}{|l|r|r|r|}
\hline \multicolumn{1}{|c|}{ Empresa } & Ene-Oct 2008 & Ene-Oct 2009 & \multicolumn{1}{c|}{$\%$} \\
\hline Topy Top & 83.1 & 75.3 & $-9.4 \%$ \\
Devanlay Perú & 104.2 & 68.3 & $-34.5 \%$ \\
Confecciones Textimax & 71.8 & 57.3 & $-20.2 \%$ \\
Textiles Camones & 35.7 & 39.9 & $11.8 \%$ \\
Cotton Knit & 33.3 & 30.1 & $-9.6 \%$ \\
Michell y Cía. & 31.4 & 29.1 & $-7.3 \%$ \\
Hilandería de Algodón Peruano & 29.1 & 26.5 & $-8.9 \%$ \\
Textil San Cristóbal & 32.6 & 26.2 & $-19.6 \%$ \\
Perú Fashions & 20.2 & 25.9 & $28.2 \%$ \\
Industrias Nettalco & 41.7 & 25.6 & $-38.6 \%$ \\
Creditex & 30.8 & 21.1 & $-31.5 \%$ \\
Industria Textil del Pacífico & 27.5 & 21.0 & $-23.6 \%$ \\
Textil del Valle & 27.8 & 20.2 & $-27.3 \%$ \\
Southern Textile Network & 25.9 & 16.0 & $-38.2 \%$ \\
Inka Designs & 5.4 & 15.8 & $192.6 \%$ \\
Inca Tops & 17.6 & 15.1 & $-14.2 \%$ \\
Cofaco & 18.5 & 14.8 & $-20.0 \%$ \\
Negocios Sudamericanos & 10.7 & 12.9 & $20.6 \%$ \\
Ideas Textiles & 22.8 & 11.8 & $-48.2 \%$ \\
Incalpaca & 15.9 & 11.5 & $-27.7 \%$ \\
Franky y Ricky & 14.2 & 10.6 & $-25.4 \%$ \\
Inka Knit & 22.9 & 10.2 & $-55.5 \%$ \\
Garment Industries & 16.0 & 8.3 & $-48.1 \%$ \\
JH Textil & 1.6 & 8.0 & $400.0 \%$ \\
Cía. Industrial Nuevo Mundo & 8.1 & 7.3 & $-9.9 \%$ \\
\hline Sub-total (25 primeras) & $\mathbf{7 4 8 . 8}$ & $\mathbf{6 0 8 . 8}$ & $\mathbf{- 1 8 . 7}$ \\
Total & $\mathbf{1 , 6 6 7 . 3}$ & $\mathbf{1 , 2 3 2 . 6}$ & $-\mathbf{2 6 . 1}$ \\
\hline
\end{tabular}

Fuente: COMEXPERU

En enero del 2009 la Sociedad Nacional de Industrias (SNI), en representación de un grupo de 10 empresas hilanderas, presentó ante la Comisión de Fiscalización de Dumping y Subsidios de INDECOPI una solicitud para la aplicación de una medida de salvaguardia general a las importaciones de hilados de algodón (partidas arancelarias 52.05 y 52.06). INDECOPI recomendó la aplicación de salvaguardias a las importaciones de las subpartidas arancelarias 520512; 520513; 520522; 520523; 520524 y 520526 , ya que durante el 2008 dichas partidas concentraron el 95.6\% del total de las importaciones de hilados de algodón en términos de volumen. Dicha institución planteó un período de aplicación no mayor a 18 meses, considerando que sería un plazo adecuado para que la economía norteamericana saliera de la recesión y volviera a dar impulso a la demanda de hilados nacionales. Señaló que los productos de dichas subpartidas son similares a los producidos localmente y estableció las equivalencias respectivas. Entre los países para los que rige la medida destaca la India, que concentró el 86.6\% del volumen importado en el 2008.

2 Ver Maximixe. (2009). Op. Cit. 


\section{MÉTODOS}

La Investigación desarrollada es de carácter exploratoria ya que realiza un análisis situacional, estudia los hechos históricos y analiza posibles escenarios mediante entrevistas a profundidad a expertos en la industria textil, así como también fuentes secundarias como estudios de entidades especializadas como Apoyo Consultoría, CENTRUM Consultoría y Maximixe, bibliografía especializada, y fuentes privadas (como la SNI) y gubernamentales como Ministerio de la Producción (PRODUCE), Instituto Nacional de Estadística e Informática (INEI), Banco Central de Reserva del Perú (BCRP), MEF, entre otros.

\section{RESULTADOS}

1. El sector textil contribuye significativamente al crecimiento del país. Se estima que en el 2010 el subsector manufacturero no primario habría crecido a la tasa interanual de $17.7 \%$, debido al avance de las ramas industriales de bienes de consumo, intermedios y de capital. La producción de bienes de consumo continuará siendo impulsada por la industria de confecciones ante la recuperación del volumen de las exportaciones de prendas y la mayor demanda interna. La producción de bienes intermedios se verá beneficiada por el avance del sector construcción ante la mayor ejecución de proyectos privados y públicos. Por su parte, la producción de bienes de capital será impulsada por la mayor demanda de vehículos para transporte público, motocicletas y aparatos eléctricos. En el último trimestre del 2010 se espera que el subsector registre menores tasas de crecimiento, debido a que estadísticamente deberá compararse con el trimestre de mayor producción del año pasado.

En agosto la manufactura no primaria creció $23.8 \%$ respecto a agosto del 2010 y acumuló un avance de $16.1 \%$ en los ocho primeros meses del año. El dinamismo del subsector estuvo determinado por la mayor producción de bienes de consumo (21.8\%), bienes intermedios (25.6\%) y bienes de capital (52\%). El avance de la producción de bienes de consumo fue determinado por la mayor producción de prendas de vestir, excepto las prendas de piel (66.6\%); tejidos y artículos de punto y ganchillo (24.7\%); artículos de papel y cartón (36.9\%); y jabones, detergentes, productos de limpieza y tocador $(28.9 \%)$, impulsados por la mayor demanda interna y externa. El avance de la producción de bienes intermedios fue explicada por la mayor actividad de impresión $(82.7 \%)$, productos metálicos para uso estructural (54.8\%), productos de arcilla y cerámica no refractaria para uso estructural $(23.4 \%)$ y cemento, cal y yeso (11.6\%), debido principalmente al avance del sector construcción y a las mayores exportaciones. El dinamismo de la producción de bienes de capital fue determinado por la mayor fabricación de vehículos automotores (404.7\%), motores, generadores y transformadores eléctricos (78.6\%), motocicletas (98.8\%) y bombas, compresores, grifos y válvulas (45.9\%).

2. El sector textil es fuente de mano de obra intensiva. Las cifras de empleo formal (en planilla) en el sector se han estabilizado en los últimos meses del 2009 (ver Cuadro N³). Según la SUNAT, en noviembre del 2009 la caída habría sido de solo 3\% respecto de los niveles de diciembre del 2008.

Cuadro $\mathbf{N}^{\circ}$ 3. Empleo en las principales empresas de textil-confecciones (Número de trabajadores en planilla)

\begin{tabular}{|l|r|r|r|}
\hline \multicolumn{1}{|c|}{ Empresa } & Ene-Oct 2008 & Ene-Oct 2009 & \multicolumn{1}{c|}{$\%$} \\
\hline Topy Top & $1,986.0$ & $2,102.0$ & $5.8 \%$ \\
Devanlay Perú & 369.0 & 356.0 & $-3.5 \%$ \\
Confecciones Textimax & $2,752.0$ & $2,373.0$ & $-13.8 \%$ \\
Textiles Camones & $1,417.0$ & $1,559.0$ & $10.0 \%$ \\
Cotton Knit & $2,317.0$ & $2,219.0$ & $-4.2 \%$ \\
Michell y Cía. & 727.0 & 756.0 & $4.0 \%$ \\
Hilandería de Algodón Peruano & $2,980.0$ & $2,256.0$ & $-24.3 \%$ \\
Textil San Cristóbal & $3,076.0$ & $3,276.0$ & $6.5 \%$ \\
Perú Fashions & $1,287.0$ & $1,381.0$ & $7.3 \%$ \\
Industrias Nettalco & 308.0 & 220.0 & $-28.6 \%$ \\
Creditex & $1,519.0$ & $1,373.0$ & $-9.6 \%$ \\
Industria Textil del Pacífico & 886.0 & $1,044.0$ & $17.8 \%$ \\
Textil del Valle & $2,717.0$ & $2,556.0$ & $-5.9 \%$ \\
Southern Textile Network & 170.0 & 139.0 & $-18.2 \%$ \\
Inka Designs & 801.0 & $1,095.0$ & $36.7 \%$ \\
Inca Tops & 764.0 & 694.0 & $-9.2 \%$ \\
Cofaco & $1,512.0$ & $1,901.0$ & $25.7 \%$ \\
Negocios Sudamericanos & $\mathrm{NA}$ & $\mathrm{NA}$ & - \\
Ideas Textiles & 485.0 & 496.0 & $2.3 \%$ \\
Incalpaca & $1,402.0$ & $1,231.0$ & $-12.2 \%$ \\
Franky y Ricky & 923.0 & 823.0 & $-10.8 \%$ \\
Inka Knit & $1,327.0$ & 936.0 & $-29.5 \%$ \\
Garment Industries & $\mathrm{NA}$ & $\mathrm{NA}$ & - \\
JH Textil & $\mathrm{NA}$ & $\mathrm{NA}$ & - \\
Cía. Industrial Nuevo Mundo & 879.0 & 858.0 & $-2.4 \%$ \\
\hline Sub-total (25 primeras) & $\mathbf{3 0 , 6 0 4 . 0}$ & $\mathbf{2 9 , 6 4 4 . 0}$ & $-3.1 \%$ \\
\hline
\end{tabular}

Fuente: SUNAT

3. El sector textil es generador de divisas para el país. Al cierre del 2010 las exportaciones del sector manufactura sumarían US\$ 4,521 millones, $18.3 \%$ más respecto al 2010. El avance proyectado de las exportaciones considera 
mayores envíos de productos químicos (38.8\%), sidero-metalúrgicos y joyería (50.1\%), minerales no metálicos (30.3\%), metalúrgicos (9.1\%), así como maderas y papeles y sus manufacturas (7.6\%). La estimación tiene como parámetro que se mantenga el buen dinamismo de las exportaciones hacia países de la región (Colombia, Chile, Ecuador, Bolivia, entre otros). Por su parte, las exportaciones de textiles y confecciones registrarían una caída de $1.2 \%$ afectadas por los problemas de los envíos hacia Venezuela. Sin embargo, si la demanda de Estados Unidos muestra mayor dinamismo en lo que resta del año, consecuencia de las acciones que viene implementado e implementará la FED, y la demanda de confecciones de Venezuela muestra signos de recuperación, las exportaciones manufactureras totales podrían alcanzar un crecimiento de $19.2 \%$ al cierre del 2010. Si por el contrario, la demanda externa del sector textil y confecciones se desacelera, el crecimiento solo alcanzaría 17.5\%.

\section{DISCUSIÓN. ANÁLISIS FODA}

Uno de los riesgos que enfrenta la industria textil es la pérdida de mercado de los hilados de algodón nacional debido a la masiva importación de hilados a menores precios. Otro riesgo es la alta dependencia de las exportaciones de la industria de confecciones, cuyo crecimiento dependerá del ritmo de la recuperación de las economías de los principales socios comerciales.

Entre las principales oportunidades están los tratados comerciales firmados con China, Canadá, Tailandia, Singapur, Unión Europea y EFTA, que permitirían la exportación libre de aranceles hacia esos países. La recuperación de la economía norteamericana y mundial impulsaría la demanda de confecciones, ya que requerirán productos textiles.

Entre las amenazas está la falta de una política de Estado para proteger la industria textil. De otro lado, la continua reducción de las áreas sembradas

Cuadro $\mathrm{N}^{\circ}$ 3. Matriz FODA del sector textil

\begin{tabular}{|c|c|}
\hline Fortalezas & Debilidades \\
\hline $\begin{array}{l}\text { - Algodón (importante insumo productivo) de primera } \\
\text { calidad con reconocimiento mundial. } \\
\text { - Adaptación de la mano de obra para acoplarse a diversos } \\
\text { escenarios cambiantes. } \\
\text { - } \text { Reconocimiento internacional por la tradición textil. } \\
\text { - Amplia capacidad instalada verticalmente integrada. } \\
\text { - } \text { Adecuado conocimiento de toda la cadena productiva } \\
\text { - Sistemas de control basados en tecnología de punta en } \\
\text { diversas etapas del proceso productivo. }\end{array}$ & 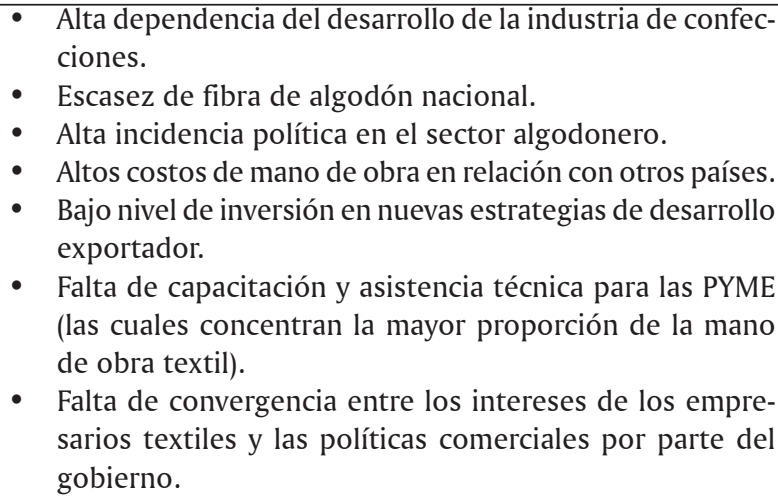 \\
\hline
\end{tabular}

Fuente: CENTRUM, Reporte Financiero, y Maximixe, Riesgos de Mercado.

3 Ver Maximixe. (2010). 
de algodón nacional, debido al incremento de la importación de los hilados, afectará el abastecimiento de algodón de primera calidad.

Finalmente, como fortalezas podemos destacar la alta calidad de nuestro algodón de fibra larga y extra larga, reconocida a nivel mundial. También, el amplio conocimiento de la cadena productiva por parte de los empresarios locales.

\section{CONCLUSIONES}

1. El sector textil peruano entró en fase de desaceleración a partir del 2009 debido a la crisis internacional, que se ha reflejado en una menor demanda internacional de los productos. Prueba de ello es la menor tasa de utilización de la capacidad instalada y el menor valor de las exportaciones. Además, en los últimos meses del 2009 se presentó una tendencia creciente en los costos de producción (hilados de algodón) que encarecen los productos finales y restan competitividad frente a los productos asiáticos, caracterizados por ser más económicos (pero de menor calidad).

2. En el 2009 las exportaciones peruanas a Estados Unidos fueron las que más retrocedieron en sus volúmenes exportados de prendas de tejido de punto de algodón. China, por su parte, continuó destacando por encima del resto.

3. Las cifras de empleo formal (en planilla) en el sector se han estabilizado en los últimos meses del 2009. Según SUNAT, en noviembre del 2009 la caída habría sido de solo $3 \%$ respecto de los niveles de diciembre del 2008.

4. La recuperación ligera de la demanda estadounidense, cierta estabilización en el mercado venezolano y una mayor presencia en otros mercados de la región, permitirán que las exportaciones del sector textil-confecciones crezcan 19\% en el 2010.

5. Los elementos de diferenciación no solo deben concentrarse en los productos sino también en el conjunto del servicio que los productores ofrecen a los compradores internacionales. Un elemento de diferenciación en el servicio, por ejemplo, es la rápida capacidad de respuesta de la industria nacional, que permite responder a pedidos de urgencia -por lo general de menor escala o de menor lote- de mejor manera que otros competidores del exterior.
6. Por último, un esfuerzo necesario y urgente será el de buscar nuevos clientes en el exterior, esfuerzo consistente con la idea de diversificar las ventas y con la posibilidad de ocuparse de pedidos de escala menores de los que se puede encargar en forma más competitiva la industria china. En el 2008, cerca del $75 \%$ de las exportaciones textiles peruanas se dirigieron a solo dos países (Estados Unidos y Venezuela).

\section{BIBLIOGRAFÍA}

\section{Libros}

DAVID, FRED. (1991). La Gerencia Estratégica. Colombia, Fondo Editorial Legis.

HITT, MICHAEL. (2004). Administración Estratégica. México, Thompson Learning.

MALHOTRA K. NARESH. (2004). Investigación de Mercados. México, Prentice Hall.

PORTER, MICHAEL. (2004). Ventaja Competitiva. México, CECSA.

\section{Documentos}

BANCO CENTRAL DE RESERVA DEL PERÚ. (2009). "Actividad Productiva y Empleo". Perú.

MINISTERIO DE ECONOMÍA Y FINANZAS DEL PERÚ. (2010). "Marco Macroeconómico Multianual 2010-2013”. Perú.

IPSOS APOYO OPINIÓN Y MERCADO. Diciembre 2010. Perú.

MAXIMIXE. Septiembre 2009. "Riesgos de mercado". Perú.

MAXIMIXE. Noviembre 2010. "Riesgos sectoriales". Perú.

CENTRUM. Septiembre 2010. "Reporte financiero". Perú.

\section{Internet}

http://www.promperu.gob.pe

http://www.mef.gob.pe/ESPEC/MMM20082010/ MMM20082010Revisado.pdf

http://www.conasev.gob.pe

http://www.bcrp.gob.pe

http://www.inei.gob.pe 